\title{
Erratum to: Extending the type reproduction number to infectious disease control targeting contacts between types
}

\author{
Zhisheng Shuai • J. A. P. Heesterbeek • P. van den Driessche
}

Published online: 24 March 2015

(C) Springer-Verlag Berlin Heidelberg 2015

\section{Erratum to: J Math Biol (2013) 67:1067-1082 DOI 10.1007/s00285-012-0579-9}

In the original publication an error in the definition of the target reproduction number $\mathcal{T}_{S}$ with respect to the target set $S$ (see Eq. (2.1) in the original paper) was recently discovered. That definition is correct if the target set $S$ consists of any number of entries from only one row (or column) of the $n \times n$ next-generation matrix $K$, or all nonzero entries from several rows (or columns) of $K$. However, it is not correct for a general target set $S$, for example, $\mathcal{T}_{U}, \mathcal{T}_{V}$ in Section 3.1, and two values of $\mathcal{T}_{S}$ in Section 5.4 of the original paper, although the stated formulas hold. A correct definition of the target reproduction number $\mathcal{T}_{S}$ is as follows.

Let $K=\left[k_{i j}\right]$ be a nonnegative irreducible $n \times n$ (next-generation) matrix. Suppose that several entries of $K$ are subject to change (target), either a decrease or increase. Let $S$ be the target set consisting of all entries subject to change, and $K_{S}$ be the target matrix corresponding to the target set $S$ defined as follows: $\left[K_{S}\right]_{i j}=k_{i j}$ if $(i, j) \in S$ and 0 otherwise. If $\rho\left(K-K_{S}\right)<1$, then the target reproduction number $\mathcal{T}_{S}$ is defined as

The online version of the original article can be found under doi:10.1007/s00285-012-0579-9.

Z. Shuai $(\varangle) \cdot$ P. van den Driessche

Department of Mathematics and Statistics, University of Victoria, Victoria, BC V8W 3R4, Canada e-mail: zshuai@uvic.ca

P. van den Driessche

e-mail: pvdd@math.uvic.ca

J. A. P. Heesterbeek

Faculty of Veterinary Medicine, University of Utrecht, Yalelaan 7, 3584 CL Utrecht, The Netherlands e-mail: j.a.p.heesterbeek@uu.nl 


$$
\mathcal{T}_{S}=\rho\left(K_{S}\left(I-K+K_{S}\right)^{-1}\right),
$$

where $I$ is the $n \times n$ identity matrix and $\rho$ denotes the spectral radius of the matrix.

In the original paper, it was misstated that the target matrix $K_{S}$ can be written as $P_{S_{1}} K P_{S_{2}}$, which thus leads to the incorrect definition as in Eq. (2.1) in the original paper. Since all derivations and calculations in the original paper utilize the correct target matrix $K_{S}$ (although stated in the form of $P_{S_{1}} K P_{S_{2}}$ ), these results and examples in the original paper remain correct, except for the typos stated below. In all places in the original paper, $P_{S_{1}} K P_{S_{2}}$ should be replaced by $K_{S}$. For example, it is stated in the proof of Theorem 2.2 in the original paper that "each targeted entry of $K$ appears only in the term $P_{S_{1}} K P_{S_{2}}$ "; here $P_{S_{1}} K P_{S_{2}}$ should be replaced by the target matrix $K_{S}$.

When the target set $S$ consists of one or more rows and all $n$ columns of $K$, the target reproduction number becomes the type reproduction number, previously defined in Heesterbeek and Roberts (2007), Roberts and Heesterbeek (2003). In particular, when $S=\{(i, j) \mid 1 \leq j \leq n\}$ for some $i$ (namely, targeting all entries in the $i$ th row of $K$ ), the target reproduction number becomes the type reproduction number denoted by $\mathcal{T}_{i}$ (Heesterbeek and Roberts 2007; Roberts and Heesterbeek 2003). When $S=\{(j, i) \mid 1 \leq j \leq n\}$ for some $i$ (namely, targeting all entries in the $i$ th column), the target reproduction number is also equal to $\mathcal{T}_{i}$ as shown in Moon et al. (2014, Section 5). In general, $K_{S}$ can consist of entries from the same row or from different rows in $K$; see Section 5 of the original paper. If $S=\{(i, j)\}$ (namely, only one entry is targeted), then the target reproduction number is customarily denoted by $\mathcal{T}_{i j}$. The formulas for $\mathcal{T}_{i j}$ and $\mathcal{T}_{i}$ stated in Eq. (2.2) and Eq. (2.3) of the original paper are correct. In addition, new expressions for $\mathcal{T}_{i j}$ and $\mathcal{T}_{i}$ are derived in Moon et al. (2014, Theorems 4.1 and 5.3) in terms of cycles in the weighted digraph associated with $K$.

The following typos and inserts for clarification in the original paper should be noted:

- Page 1071 , line -1 : the $(1,2)$ entry in the matrix is $k_{12} /\left(1-k_{22}\right)$ rather than 0 , but the stated result for $\mathcal{T}_{S}$ holds.

- Page 1072, line -1: the coefficient for the product $k_{11} k_{12} k_{21} k_{22}$ in $\mathcal{T}_{V}$ should be 4 rather than 1.

- Page 1073, line -11: insert "and this arc is the last arc of the walk" before the end of the sentence.

- Page 1073, line -1: insert "and this arc is the last arc of the walk" before "but no other arcs".

- Page 1074, line 7: insert "and this arc is the last arc of the walk" before the end of the sentence.

In addition the authors would like to present the current affiliation and email, for communication purpose as given below.

Zhisheng Shuai

Department of Mathematics, University of Central Florida Orlando, FL 32816, USA

email: shuai@ucf.edu 


\author{
J. A. P. Heesterbeek \\ Faculty of Veterinary Medicine, University of Utrecht Yalelaan 7, 3584 CL, \\ Utrecht, The Netherlands \\ email:j.a.p.heesterbeek@uu.nl \\ P. van den Driessche \\ Department of Mathematics and Statistics, University of Victoria \\ Victoria, BC V8W 2Y2, Canada \\ email:pvdd@math.uvic.ca
}

Acknowledgments The authors wish to thank Professors James Watmough and John Moon for inquiring about the definition of $\mathcal{T}_{S}$ in the original paper.

\title{
References
}

Heesterbeek JAP, Roberts MG (2007) The type-reproduction number $T$ in models for infectious disease control. Math Biosci 206:3-10

Moon JW, Shuai Z, van den Driessche P (2014) Walks and cycles on a digraph with application to population dynamics. Linear Algebra Appl 451:182-196

Roberts MG, Heesterbeek JAP (2003) A new method for estimating the effort required to control an infectious disease. Proc R Soc Lond B 270:1359-1364 